\title{
ANÁLISIS DE REDES SOCIALES COMO POSIBILIDAD TEÓRICO- METODOLÓGICA PARA LA INVESTIGACIÓN EDUCATIVA
}

SOCIAL NETWORK ANALISYS AS THEORICAL AND METODOLOGYCAL POSSIBILITY FOR THE EDUCACTIONAL RESEARCH

\author{
Volumen 11, Número 3 \\ Setiembre-Diciembre
}

pp. 1-15

Este número se publicó el 15 de diciembre de 2011

Luis Carlos Morales Zúñiga

La revista está indexada en los directorios:

LATINDEX, REDALYC, IRESIE, CLASE, DIALNET, DOAJ, E-REVIST@S,

La revista está incluida en los sitios:

REDIE, RINACE, OEI, MAESTROTECA, PREAL, HUASCARAN, CLASCO 


\section{ANÁLISIS DE REDES SOCIALES COMO POSIBILIDAD TEÓRICO- METODOLÓGICA PARA LA INVESTIGACIÓN EDUCATIVA \\ SOCIAL NETWORK ANALISYS AS THEORICAL AND METODOLOGYCAL POSSIBILITY \\ FOR THE EDUCACTIONAL RESEARCH}

\section{Luis Carlos Morales Zúñiga ${ }^{1}$}

Resumen: El artículo tiene como principal objetivo sugerir la utilización de la Teoría de Redes Sociales para la investigación educativa. Para ello, inicialmente se señala el origen y la definición de la Teoría de Redes Sociales; después, se presentan tres ejemplos de análisis de redes sociales en la investigación social. Por último, se proponen algunos lineamientos para utilizar la Teoría de Redes Sociales en la investigación educativa.

Palabras clave: REDES SOCIALES, INVESTIGACION EDUCATIVA, INVESTIGACION SOCIAL, EDUCACION, SOCIEDAD.

Abstract: The article's main objective is to suggest the use of Social Network Theory for educational research. To do this, initially noting the origin and definition of Social Network Theory, then presents three examples of social network analysis in social research in general. Finally we propose some guidelines for using social network theory in educational research.

Keywords: SOCIAL NETWORKS, EDUCATIONAL RESEARCH, SOCIAL RESEARCH, EDUCATION, SOCIETY.

\footnotetext{
${ }^{1}$ Magíster Scientiae en Sociología por la Maestría Centroamericana en Sociología con sede en la Universidad de Costa Rica (UCR). Licenciado en la Enseñanza de los Estudios Sociales y la Educación Cívica. Profesor de la Escuela de Formación Docente de la Universidad de Costa Rica Profesor de la Maestría en Ciencias de la Educación, con mención en Administración Educativa, de la Universidad de Costa Rica.
}

Dirección electrónica: luis23m@gmail.com

Artículo recibido: 15 de julio, 2010

Aprobado: 28 de marzo, 2011 


\section{Notas introductorias ${ }^{2}$}

La investigación educativa ha obtenido enormes aportes, tanto teóricos, como metodológicos, e interpretativos, del resto de las Ciencias Sociales, sobre todo, si se parte del supuesto de que la educación es un hecho sociaß por excelencia, y como hecho social, debe ser estudiado con la rigurosidad de la Ciencia Social.

Es posible reconocer ejemplos de la utilización de numerosos corpus teóricos, o bien, de múltiples recursos metodológicos que provienen de las Ciencias Sociales y que han sido aplicados para comprender y explicar la realidad educativa, en sus múltiples aristas, o bien, intervenir sobre ella con tal de transformar o mejorar algún aspecto particular.

En ese sentido, una revisión rápida y general de la investigación realizada en el ámbito de la educación, nos podría llevar a investigaciones educativas que han empleado modelos teórico-metodológicos diversos. Desde la hermenéutica, hasta la teoría crítica, el estructuralismo, la fenomenología, la etno-metodología, la teoría de las representaciones sociales, el análisis de discurso y la investigación-acción, por hablar tan solo de algunos aparatos teóricos utilizados. $Y$ en el ámbito metodológico, claramente la utilización de enfoques cualitativos, cuantitativos, mixtos, y la subsecuente derivación técnica de cada enfoque seleccionado.

La investigación educativa, entonces, es tributaria de la investigación en Ciencias Sociales; sin embargo, es importante resaltarlo, con tal de no desligar el desarrollo de la ciencia social y su correlato representado, en este caso, por el desarrollo de la investigación en el campo de la educación.

A pesar de lo anterior, la corriente teórico-metodológica conocida como Teoría de Redes Sociales, cuyo origen se encuentra en la Sociología, ha tenido muy poca utilización como marco de referencia en la investigación educativa. Al menos, en el ámbito de la investigación realizada a nivel de grado y de posgrado y de institutos de investigación en la Universidad de Costa Rica no existen, en este momento, registros de investigaciones que hayan utilizado la Teoría de Redes Sociales para el análisis de algún fenómeno educativo.

2 Una versión inicial de este documento fue presentada como ponencia en el II Congreso de Educación Primaria. Investigar e Innovar: Retos de la Educación Primaria, Universidad de Costa Rica, julio de 2010.

3 El concepto de hecho social se entiende en el sentido que le da Emile Durkheim (2001), como un conjunto de maneras de obrar, de pensar y de actuar que son exteriores al individuo, y que necesariamente están cargadas de un poder coactivo que les permite imponerse. Por consiguiente, -dice Durkheim- un hecho social es toda manera de hacer, fijada o no, susceptible de ejercer sobre el individuo una coacción exterior (pp.26-27). 
Incluso, el desarrollo de la Sociología de la educación en Costa Rica, no pasa por la aplicación de esta teoría a la investigación en educación. Una posible explicación a esta ausencia podría ser el poco desarrollo de la sociología de la educación en nuestro país ${ }^{4}$, lo cual claramente implica un limitado abordaje teórico-metodológico de los objetos de estudio que ha investigado.

En este marco de condiciones, el objetivo principal de este artículo es plantear cuáles son las posibilidades de la Teoría de Redes Sociales para el estudio de algunos fenómenos educativos, con tal de sugerir su utilización.

Para alcanzar este propósito, en el primer apartado se esboza, de manera general, el origen y la definición de la Teoría de Redes Sociales, para entender de qué se trata esta teoría. Posteriormente, se reseñan tres casos de investigación social, donde se ha utilizado la Teoría de Redes Sociales como marco de referencia, para concluir con un intento de dar respuesta a la pregunta: ¿Cómo se podría utilizar la Teoría de Redes Sociales en la investigación educativa? La respuesta a esta interrogante nos permitirá plantear, entonces, el análisis de redes sociales como un potencial marco teórico-metodológico para la investigación educativa, lo cual es una sugerencia y, además, una invitación.

\section{Teoría de Redes Sociales: origen y definición}

Como quedó planteado, el origen de la Teoría de Redes Sociales es estrictamente sociológico, y se encuentra, en específico, en uno de los autores clásicos de la sociología, George Simmel, (1858-1918). Para este sociólogo alemán, el tema del cruce de los círculos sociales es de importancia capital para la comprensión de lo real-social, pues el observador debe hacer evidentes las ligaduras que existen en las formaciones sociales, las cuales no son apreciables mediante la observación superficial, pues se encuentran ocultas tras la apariencia de un todo unitario, o bien de la simple representación casual. Por lo tanto, la ciencia social, en general y particularmente la sociología, necesita reconstruir las formas de las unidades sociales, con tal de lograr aprehender el contenido de las representaciones que está inmerso en tales formas. Respecto a lo anterior, Simmel (1939) plantea lo siguiente

\footnotetext{
4 Tal como lo habíamos expuesto en un artículo anterior, titulado Balance de la Sociología de la Educación en Costa Rica (Morales, 2010).
} 
Al principio basta la coincidencia casual en el espacio y en el tiempo para que se asocien psicológicamente las representaciones. La reunión de propiedades que constituye un objeto concreto, aparece primero como un todo unitario, y cada una se halla en estrecha conexión asociativa con aquellas otras en cuya proximidad ha estado siempre vista... Entonces percibimos claramente lo que hay de igual en todas estas representaciones, y al propio tiempo las vemos recíprocamente ligadas, las aprehendemos cada vez más libre de su convivencia meramente casual con el objeto al que primeramente las habíamos visto unidas. (p. 7)

En este sentido, es Simmel uno de los primeros que llama la atención sobre las posibilidades epistémicas y teóricas del análisis de las ligaduras y las conexiones entre los individuos que en última instancia conforman la sociedad. Desde esta perspectiva el investigador se encarga de reconstruir las formas (redes) que se encuentran invisibles en una estructura social determinada, con el objetivo de acceder a los contenidos que encierran tales formas.

El investigador le imputa sentido a las relaciones e interacciones o, más bien, en términos de Simmel, a las acciones recíprocas que se dan en un contexto espacial y temporal determinado, y efectúa una operación científica que permite ver más allá de lo aparente, reconstruir las formas de lo social, y conseguir el acceso a los contenidos.

Simmel plantea que los cruces de los círculos sociales y las acciones recíprocas adquieren una suerte de naturalización para las conciencias individuales, puesto que se dan en función de las condiciones del lugar social de los individuos, condiciones que no han sido necesariamente optadas por ellos, de igual manera como Karl Marx señalaba enfáticamente que los individuos hacen su historia en condiciones heredadas.

Por lo tanto, las acciones recíprocas se dan en referencia a las condiciones inmediatas de los agentes sociales, los cuales construyen o tejen sus acciones recíprocas, basándose en aspectos como la colaboración, la solidaridad, la competencia, la exclusión o la inclusión, y les imputan contenidos y representaciones particulares dando origen a las formaciones sociales

El individuo se ve primeramente colocado en un medio para el cual su individualidad es relativamente indiferente, medio que le encadena a su propio destino y le impone una estrecha convivencia con aquellos junto a quienes le ha situado el azar del nacimiento. 
Este adverbio primeramente significa el estadio inicial, tanto de la evolución filogenética, como de la ontogenética. (Simmel, 1939, p. 13)

Se sugiere, por ende, la pertinencia de la reconstrucción de los entramados de relaciones recíprocas que se desarrollan entre las distintas individualidades. Es en tales redes de acciones recíprocas, donde se pone en evidencia las características de las formaciones sociales y los contenidos de esas formaciones.

Precisamente, el tema de la acción recíproca es en Simmel fundamental para lograr la captación de las realidades sociales, o de las estructuras sociales, al punto que la sociedad existe cuando existen acciones recíprocas, lo cual lleva a la centralidad que Simmel (1939) le confiere a la interacción social

La sociedad existe allí donde varios individuos entran en acción recíproca. Esta acción recíproca se produce siempre por determinados instintos o por determinados fines. Instintos eróticos, religiosos, o simplemente sociales, fines de defensa o de ataque, de juego o de adquisición, de ayuda o de enseñanza, e infinitos otros, hacen que el hombre se ponga en convivencia, en acción conjunta, en correlación de circunstancias con otros hombres; es decir, que ejerza influencias sobre ellos, y a la vez las reciba de ellos. La existencia de estas acciones recíprocas significa que los portadores individuales de aquellos instintos, y fines, que los movieron a unirse se han convertido en una unidad, en una sociedad. (p.13)

Para Simmel, es evidente que las acciones recíprocas son los elementos confieren unidad a las formaciones sociales, pues para que exista la unidad se necesita que se den una serie de influencias mutuas entre los distintos componentes.

Entonces, en una red social hay características importantes, por ejemplo, el tema de la acción recíproca y la interacción entre diversos elementos que adquieren una unidad propia, que si bien no es perceptible mediante la simple observación, sí se puede identificar y reconstruir a través de la investigación. No obstante, en este punto es importante responder a la pregunta, ¿cómo podría definirse una red social? Hay múltiples definiciones de lo que una red social es.

Para el sociólogo francés Pierre Merkclé, quien analiza los planteamientos de Simmel con tal de continuar la construcción de la Teoría de Redes Sociales, una red social se traza 
de forma espontánea mediante flujos de información y de relaciones que se dan de maneras conscientes o inconscientes y que tienen un objetivo determinado. En tal sentido, Pierre Mercklé (2004) aporta una definición de red social, que resulta de interés para la investigación en Ciencias Sociales en general

Un réseau social, dans cette perspective, peut être ici défini provisoirement comme constitué d'un ensemble d'unités sociales et des relations que ces unités sociales entretienent les unes avec les autres, directement, ou indirectement, à travers des chaînes de longueurs variables. Ces unités sociales peut être des individus, des grupes informels d'individus ou bien des organisations plus formelles ${ }^{5}$. (p.4)

Como se puede observar, Mercklé pone un especial interés no solo en las unidades sociales que integran una determinada red, sino que, resalta además el tipo de relaciones que tales unidades sociales mantienen recíprocamente, y que pueden tener una variable formación.

Otra definición interesante es la que aporta Carlos Lozares, (2008, p. 5) para quien una red social puede definirse de la siguiente forma: "Es un conjunto bien definido de actoresindividuos, grupos, organizaciones, comunidades, sociedades globales, etc., que están vinculados unos a otros a través de una o un conjunto de relaciones sociales".

Por lo tanto, una red social podría definirse como un agregado de individuos o actores sociales que entran en relación debido a múltiples motivaciones, las cuales están insertas en la estructura social, y en función de ella, pues estos forman parte de la estructura y además la constituyen. En ese sentido, la acción social está motivada por las necesidades y las demandas que la estructura plantea al individuo, de ahí el carácter estructuralista de la Teoría de Redes Sociales. No obstante, ese carácter estructuralista es matizado. Burt, citado por Lozares, (2008) plantea la Teoría de las Redes Sociales en una versión estructuralista no tradicional

\footnotetext{
5 Una red social, en esta perspectiva, puede ser definida provisoriamente como constituida por un conjunto de unidades sociales y de relaciones que esas unidades sociales mantienen las unas con las otras, directa 0 indirectamente, a través de cadenas de longitudes variables. Esas unidades sociales pueden ser individuos, grupos informales de individuos, o bien organizaciones formales (traducción propia).
} 
El desarrollo que Burt, Burt R.S., 1982, hace de las Redes como aproximación estructural difiere evidentemente de una visión normativa y atomista. Parte de la existencia de un conjunto estatus/roles de los actores generados por la división de trabajo. Un actor evalúa la utilidad de las acciones alternativas en parte en función de las condiciones personales y en parte en función de las condiciones de los otros: extensión de la concepción atomista y restricción de la normativa.

De esta manera, los intereses del actor social motivan acciones que están dadas en un marco contextual que es la estructura social, dentro de la cual se lleva a cabo la acción. Claro, haciendo la salvedad de que, probablemente, desde la teoría estructuralista francesa, Pierre Bourdieu (1930-2002) corregiría los términos actor/acción por agente/prácticas, ya que la noción de actor en el estructuralismo francés de corte bourdesiano, posee un margen de acción mucho mayor que el agente.

Las relaciones sociales son fenómenos de enorme complejidad y es de este complejo conjunto de relaciones que surgen las redes sociales y, sobre todo, los vínculos y las posiciones que los individuos toman en tales redes. $Y$ es necesario, además, considerar que esos vínculos y posiciones, así como formas de pensar, actuar y hacer, se ven modificadas por la misma pertenencia a la red social. Al respecto, Luis Sanz (2003) anota otra definición muy interesante de la Teoría de Redes Sociales

Las redes sociales son a la vez la causa y el resultado de las conductas de los individuos. Las redes sociales crean y limitan las oportunidades para la elección individual y de las organizaciones; al mismo tiempo los individuos y organizaciones inician, construyen, mantienen y rompen las relaciones y, a través de estas acciones, determinan y transforman la estructura global de la red. (p.26)

En el sentido de Luis Sanz, es importante tener en cuenta que el análisis de redes sociales no solo implica la captación y la descripción de las relaciones en las que se encuentran diversos agentes sociales, (lo cual ya es importante), pues involucra, además, comprender algunas de las oportunidades y las limitaciones a las que esos agentes se enfrentan $y$, en consecuencia, comprender el sentido que tienen las formas de hacer, de pensar y de actuar de los individuos. 
Asimismo, es necesario resaltar y advertir que el análisis de redes sociales no es solamente la simple descripción de la estructura de la red, sino que se trata de captar el funcionamiento de la red social, como el resultado de hechos sociales mayores.

En última instancia, la red es el resultado de las condiciones en las cuales los individuos se mueven y establecen sus relaciones, motivados tanto por intereses individuales como por las condiciones que la estructura social plantea, y el capital que está en juego en tales condiciones y tal estructura.

Sobre esta posibilidad de comprensión de lo real-social, que permite el análisis de redes sociales, Mercklé (2004, p. 93) lo plantea de la siguiente manera

L'analyse des réseaux n'est pas une technique visant simplement la description des structures sociales, une sorte de "sociographie" du monde social. Elle part en effet d'un postulat clasique, celui de la dimensión coercitive des phénomènes sociaux, qui définit l'approche sociologique depuis Durkheim. Mais elle specifie ce postulat en recherchant les causes des faits sociaux dans les caracteristiques des environnements structuraux dans lesquels ils s'insèrent. La forme des réseaux peut être prise comme facteur explicatif des phenomènes sociaux analysés, parce que, par exemple, elle determine l'accessibilité de certaines ressources sociales comme le prestige, la amitié, le pouvoir 6 .

Por ende, se puede apreciar cómo el análisis de redes sociales no es solamente descriptivo, sino que puede ser también explicativo y permite comprender mejor, la realidad social que sea de interés analizar, con tal captar el funcionamiento de fenómenos sociales específicos.

\footnotetext{
6 El análisis de redes no es una técnica simplemente para la descripción de las estructuras sociales, una suerte de "socio-grafía" del mundo social. Tal análisis parte en efecto de un postulado clásico, el de la dimensión coercitiva de los fenómenos sociales, que define el enfoque sociológico desde Durkheim. Sin embargo, especifica ese postulado en la investigación de las causas de los hechos sociales, en las características de los entornos estructurales en los cuales se inserta. La forma de la red puede ser tomada como factor explicativo de los fenómenos sociales analizados, ya que, por ejemplo, la red determina la accesibilidad a ciertos recursos sociales como el prestigio, la amistad, el poder... (traducción propia).
} 


\section{¿Cómo se ha utilizado la Teoría de Redes Sociales en la investigación social en la Universidad de Costa Rica? ${ }^{7}$}

En este apartado, el objetivo es mostrar, brevemente, las únicas tres investigaciones que han utilizado las posibilidades teóricas y metodológicas de la Teoría de Redes Sociales, como parte central del trabajo investigativo.

Estas investigaciones han sido llevadas a cabo como trabajos finales de graduación en la Universidad de Costa Rica (UCR), constituyen aportes importantes a las ciencias sociales y, además, ponen en evidencia la utilidad de la Teoría de Redes Sociales, aportando pistas para la investigación educativa.

En primera instancia, se encuentra la investigación de Orlando Guevara (2004), titulada: Dinámica microsocial del suicidio en adolescentes, un estudio de redes sociales. Este trabajo, Guevara se adentra en la comprensión de los fenómenos que son exteriores al sujeto y que finalmente propician el desencadenamiento de un proceso interno: el suicidio.

En esta investigación el recurso de la Teoría de Redes Sociales ha sido utilizado con tal de describir el entorno inmediato del sujeto, así como la calidad y la cantidad de las relaciones de adolescentes que sobrevivieron a un intento de suicidio.

Uno de los aportes interesantes en esta investigación tiene que ver con la posibilidad de conocer las redes familiares y de amigos en las cuales estaban insertos los adolescentes que intentaron suicidarse, reconstruyendo la fuerza de los vínculos que cada adolescente tenía con el resto de integrantes de la red, la densidad de la red (cantidad de nodos o sujetos que la componen) y la integración en cada red social.

De esta manera, Guevara logra captar las relaciones de contención y de apoyo que producen las redes sociales de adolescentes, estudiando específicamente cuatro casos de sujetos entre los 14 y los 18 años que tomaron la decisión de suicidarse sin conseguirlo. En cada caso, esta investigación estudió las relaciones que los individuos mantenían con cada uno de los miembros de la red familiar, y de la red de amigos, para concluir identificando los factores micro-sociales que propiciaron, en cada persona, tomar la decisión de autoeliminarse.

\footnotetext{
${ }^{7}$ Las tres investigaciones que reseñamos a continuación han sido dirigidas por el sociólogo, Dr. Oscar Fernández González, quien ha dado un importante impulso al desarrollo de este tipo de investigaciones en la Universidad de Costa Rica.
} 
La segunda investigación realizada en nuestro país utilizando la teoría y la metodología del análisis de redes sociales, se titula: Migraciones de nicaragüenses hacia Costa Rica: análisis de dos redes sociales, la cual fue una investigación desarrollada por Dalia Borge Marín (2004).

En este trabajo, Dalia Borge se propuso estudiar el fenómeno de la migración nicaragüense, mediante la reconstrucción y el análisis de las redes sociales que establecen aquellos que se proponen desplazarse desde Nicaragua hacia Costa Rica con tal de obtener trabajo.

En este caso, Borge analizó las relaciones entre miembros de unidades domésticas, (tanto en el sector de servicios como en el agrícola) y los miembros de unidades domésticas de nicaragüenses que viven en Costa Rica, con el fin de responder a la pregunta, ¿cómo obtienen trabajo los nicaragüenses en Costa Rica?

De esta manera, el trabajo investigativo de Borge logra aclarar, en mucho, el proceso migratorio de trabajadores nicaragüenses y su inserción en el mercado laboral de Costa Rica. Uno de los aportes del estudio en cuestión, ha sido la posibilidad de observar cómo los trabajadores nicaragüenses pueden encontrar trabajo desde su país de origen, mediante la red social a la que pertenecen, y mediante los vínculos, tanto fuertes como débiles, pero sobre todo a través de los débiles, confirmando la tesis central de Mark Granovetter ${ }^{8}$ (1973), donde se plantea que las conversaciones informales, el encuentro no planeado con personas conocidas con las que se tiene poca interacción, o bien, relaciones casuales pueden, en algún momento, jugar un papel relativamente determinante, en este caso, convertirse en un contacto que permite conseguir empleo.

Para terminar, el tercer estudio realizado en nuestro país utilizando la Teoría de Redes Sociales, es la tesis propuesta por el autor de este artículo realizada en el Programa Centroamericano de Maestría en Sociología, que lleva por título: De las actividades económicas tradicionales al auge del turismo en el cantón de Santa Cruz, Gte: el desplazamiento de la fuerza laboral hacia el sector turístico, presentada en el primer semestre de 2010.

8 En su célebre estudio titulado La fuerza de los lazos débiles, Mark Granovetter logra demostrar la importancia que tienen algunas relaciones intermitentes, o superficiales, que sostienen los individuos de manera casual y esporádica, y que facilitan el proceso de obtención de trabajo. 
En esta investigación partimos del supuesto de que al darse una reestructuración de la dinámica económica y productiva en el país, y también en la provincia de Guanacaste, (donde se ha incentivado de múltiples maneras el desarrollo del sector turístico, en detrimento de la economía tradicional, fundamentalmente de la ganadería, la agricultura y la pesca) algunos trabajadores han tenido que desplazarse hacia el sector turístico con tal de poder conseguir trabajo, puesto que las antiguas actividades han sido desarticuladas. En este sentido, se intentó captar la relación entre biografía e historia, y además, mediante la Teoría de Redes Sociales, identificar, reconstruir, e interpretar una red social de desplazamiento laboral.

En el trabajo se trata de evidenciar lo que ha ocurrido con esos trabajadores que se han desplazado de actividad, cómo ha sido el proceso de desplazamiento de actividad laboral y qué consecuencias ha tenido tal desplazamiento en las condiciones de vida y la identidad laboral de estos trabajadores.

Además, se construyó una tipología del desplazamiento de trabajadores hacia el sector turístico, tomando en cuenta los motivos por los cuales han decidido trabajar en el turismo, la forma en cómo se han desplazado, si ha sido total o parcial, la identificación hacia las nuevas y las viejas actividades, así como las consecuencias de tal desplazamiento, esto con el objetivo de generar un cuadro explicativo del objeto de estudio.

Tal como se puede apreciar, en esta breve presentación de las investigaciones en Ciencias Sociales que han utilizado la Teoría de Redes Sociales, su aplicación puede ser efectuada para el análisis de temas diversos, siendo una perspectiva novedosa y que enfoca los distintos objetos de estudio desde otro ángulo.

Cada uno de los temas tratados por las investigaciones reseñadas, suicidio en adolescentes, inmigración nicaragüense y turismo, son áreas temáticas que han sido abordadas desde diversos ángulos, pero en cada investigación, la Teoría de Redes Sociales permitió realizar análisis que enfocaran otras aristas del problema, otro tipo de información, lo cual sugiere que su utilización en la investigación educativa podría aportar nuevas visiones de diversas áreas temáticas. 


\section{Algunas consideraciones finales: ¿Cómo se podría utilizar la Teoría de Redes Sociales en la investigación educativa?}

Dar una respuesta a la pregunta planteada es una tarea de alta dificultad, pues en buena medida, el análisis de redes sociales depende no solo de los intereses del investigador, sino que además está en función del objeto de estudio definido, los recursos metodológicos complementarios, e incluso la creatividad del investigador, aspecto que no debe dejarse de lado en la investigación en general.

Dicho lo anterior, este apartado final, no busca en modo alguno ser una suerte de manual del análisis de redes sociales y su aplicabilidad a la investigación educativa, sino que, siendo menos pretencioso, el objetivo es plantear algunas líneas generales que puedan orientar a aquellos que se interesen en utilizar este valioso recurso teórico-metodológico.

En este momento, es importante retomar una de las ideas que se ha planteado en la introducción de este documento, donde se sugería que la educación es un hecho social por excelencia, de lo cual se sigue que existen elementos del fenómeno educativo que podrían ser captados mediante la Teoría de Redes Sociales.

Para alcanzar un mayor nivel de concreción, se proponen, entonces, las siguientes sugerencias operativas para el análisis de redes sociales en investigación educativa:

1. Lo primero es identificar y definir un objeto de estudio dentro del contexto educativo que sea realmente abordable mediante la Teoría de Redes Sociales, esto es, un objeto que implique distintas relaciones sociales, y que la captación y descripción de esas relaciones permitan comprenderlo mejor, y dar una explicación ante el problema y las preguntas de investigación planteadas. Es necesario clarificarse si es pertinente un análisis de redes sociales, pues dentro de las ciencias de la educación, no todos los fenómenos pueden ser abordados y explicados utilizando categorías de la Teoría de Redes Sociales, ya que existen cuestiones de orden didáctico, pedagógico o metodológico que son explicables desde otros marcos de referencia teóricometodológicos.

2. Es indispensable definir el carácter de la red social que se busca analizar. Como se observó en las investigaciones reseñadas, en cada una se definieron distintos tipos de redes, la primera investigación definió redes familiares y de amistad, establecidas 
por adolescentes suicidas; en la segunda, se definió una red de inmigración laboral de nicaragüenses hacia Costa Rica, finalmente en la tercera, una red de desplazamiento laboral desde actividades tradicionales hacia el turismo.

Este punto es fundamental, porque de otra manera no se podría tener claro el tipo de red social que se analiza, y de esta claridad se debe desprender el tipo de información que se busca obtener y analizar. En el caso de la investigación educativa existe una amplia gama de posibilidades, redes de amistad, redes de solidaridad, redes de deserción, redes de éxito, entre otras. En fin, esta definición dependerá de los intereses y la creatividad del investigador.

3. Definir los sujetos que se encuentran en el centro de la red. Es necesario reconocer el o los sujetos centrales, puesto que ellos son los que guiarán al investigador hacia los otros sujetos que conforman la red, y finalmente reconstruir la red social (tarea fundamental del análisis de redes sociales).

4. Plantearse preguntas en torno a la influencia de redes extra-escolares, sobre el desarrollo de sujetos o procesos educativos. En algunos casos, existen relaciones que se dan fuera de las instituciones educativas, y que tienen alguna o varias implicaciones sobre los que se encuentran dentro de la institución. Estas relaciones pueden ser rastreadas, reconstruidas y analizadas mediante la Teoría de Redes Sociales, y plantear así formas de intervención.

5. Es importante tener en cuenta que el análisis de redes sociales no solo es aplicable a lo microsocial y a las relaciones entre sujetos, también es aplicable a relaciones entre instituciones, y entre estas y los sujetos. De modo que la investigación educativa puede valerse de la Teoría de Redes Sociales con tal de comprender no solo las relaciones de aula, o bien, las relaciones que se dan dentro de la institución, es decir, de lo microsocial, sino que es posible proponerse la comprensión de lo macro social, como las relaciones entre diversos actores sociales que intervienen sobre la educación, instituciones (locales, nacionales, internacionales), sindicatos, entes gubernamentales o no gubernamentales, entre otros. 
6. Complementar el análisis de redes sociales con otros recursos metodológicos. Es importante, y deseable, que el análisis de redes sociales vaya acompañado de la utilización de otros recursos metodológicos tales como entrevistas, cuestionarios, grupos focales, historias de vida, observación, entre otros, siempre teniendo en cuenta el carácter de la red. Esto es fundamental, puesto que permite profundizar en el análisis de la red o redes sociales que se busquen estudiar, y realizar no solo una descripción de la estructura de la red, sino, además, comprender su funcionamiento y las relaciones que se dan en ella.

7. La utilización de software para el análisis de redes sociales también es un recurso metodológico importante. Existen varios programas que permiten analizar redes sociales, los cuales son de fácil utilización y además son software libre. Uno de ellos es el programa AGNA, que permite mediante una interfaz sencilla, graficar redes sociales, a partir de la información recopilada en el campo y observar las distintas relaciones y posiciones de los sujetos en la red de estudio.

Por último, es importante destacar que la investigación educativa podría tener en la Teoría de Redes Sociales, un recurso valioso y útil, que permitiría abordar los más diversos fenómenos educativos, desde una perspectiva novedosa. Una investigación que utilice este recurso teórico-metodológico vendría a ser pionera en la investigación educativa, y podría al mismo tiempo, marcar un prolífico comienzo para el análisis de redes sociales en distintas temáticas relacionadas con los fenómenos socioeducativos.

\section{Referencias}

Borge Marín, Dalia. (2004). Migraciones de nicaragüenses hacia Costa Rica: análisis de dos redes sociales. Tesis sometida a la consideración de la Comisión del Programa de Estudios de Postgrado en Gobierno y Políticas Públicas para optar por el grado de Doctor. Universidad de Costa Rica, San José, Costa Rica.

Granovetter, Mark S. (1973).The strength of weak ties. American Journal of Sociology, 78, (6), $1360-1380$.

Guevara Villalobos, Orlando. (2004). Dinámica microsocial del suicidio en adolescentes, un estudio de redes sociales. Tesis para optar por el grado de Licenciatura en Sociología. Escuela de Antropología y Sociología, Facultad de Ciencias Sociales, Universidad de Costa Rica, San José, Costa Rica. 
Lozares, Carlos. (1996). La Teoría de Redes Sociales. Recuperado de http://ddd.uab.cat/pub/papers/02102862n48/02102862n48p103.pdf.

Mercklé, Pierre. (2004). Sociologie des réseaux sociaux. París: Éditions La Découvert \& Syros.

Morales Zúñiga, Luis Carlos. (2010). Balance de la sociología de la educación en Costa Rica. Revista Reflexiones, Facultad de Ciencias Sociales, 89(1), Universidad de Costa Rica.

Morales Zúñiga, Luis Carlos. (2010). De las actividades económicas tradicionales al auge del turismo en el cantón de Santa Cruz, Gte: el desplazamiento de la fuerza laboral hacia el sector turístico. Tesis sometida a la consideración de la Comisión del Programa de Estudios de Posgrado en Sociología para optar al grado de Magister Scientiae en Sociología. Ciudad Universitaria Rodrigo Facio, San José.

Sanz Menéndez, Luis. (2003). Análisis de Redes Sociales: o cómo representar las estructuras sociales subyacentes. Apuntes de Ciencia y Tecnología, (7), Unidad de Políticas Comparadas, Consejo Superior de Investigaciones Científicas C/ Alfonso XII, 18, 28014 Madrid.

Simmel, George. (1939). Sociología. Estudios sobre las formas de socialización (Tomo II). Buenos Aires: Espasa-Calpe S.A. 